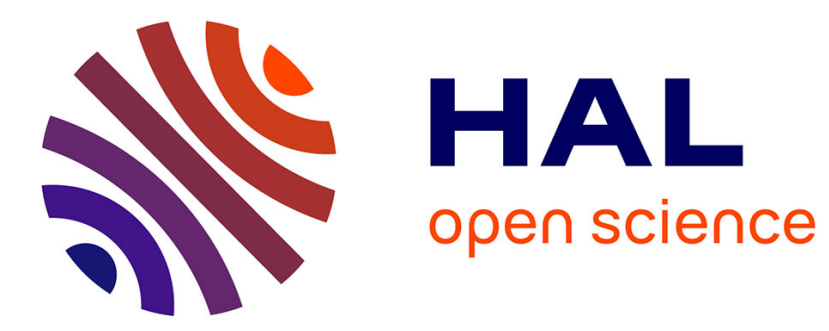

\title{
Temperature dependence of small polaron population decays in iron-doped lithium niobate by Monte Carlo simulations
}

\author{
Imed Mhaouech, Laurent Guilbert
}

\section{To cite this version:}

Imed Mhaouech, Laurent Guilbert. Temperature dependence of small polaron population decays in iron-doped lithium niobate by Monte Carlo simulations. Solid State Sciences, 2016, 60, pp.28 - 36 . 10.1016/j.solidstatesciences.2016.08.002 . hal-01562382

\section{HAL Id: hal-01562382 \\ https://hal.science/hal-01562382}

Submitted on 19 Jul 2017

HAL is a multi-disciplinary open access archive for the deposit and dissemination of scientific research documents, whether they are published or not. The documents may come from teaching and research institutions in France or abroad, or from public or private research centers.
L'archive ouverte pluridisciplinaire HAL, est destinée au dépôt et à la diffusion de documents scientifiques de niveau recherche, publiés ou non, émanant des établissements d'enseignement et de recherche français ou étrangers, des laboratoires publics ou privés. 


\title{
Temperature dependence of small polaron population decays in iron- doped lithium niobate by Monte Carlo simulations
}

\author{
I. Mhaouech*, L. Guilbert \\ Laboratoire Matériaux Optiques, Photonique et Systèmes, Université de Lorraine et CentraleSupélec, 2 rue E. Belin, Metz, France
}

\section{A R T I C L E I N F O}

\section{Article history:}

Received 22 February 2016

Received in revised form

27 July 2016

Accepted 2 August 2016

Available online 4 August 2016

\section{Keywords:}

Lithium niobate

Photorefraction

Hopping transport

Polarons

Light-induced absorption

\begin{abstract}
A B S T R A C T
The population decay of light-induced small polarons in iron-doped lithium niobate is simulated by a Monte-Carlo method on the basis of Holstein's theory. The model considers random walks of both bound polarons $\left(\mathrm{Nb}_{\mathrm{Li}}^{4+}\right)$ and free polarons $\left(\mathrm{Nb}_{\mathrm{Nb}}^{4+}\right)$ ending to deep traps $\left(\mathrm{Fe}_{\mathrm{Li}}^{3+}\right)$. The thermokinetic interplay between polaron species is introduced by trapping and de-trapping rates at niobium antisites $\left(\mathrm{Nb}_{\mathrm{Li}}\right)$. The decay of the $\mathrm{Nb}_{\mathrm{Li}}^{4+}$ population proceeds by three possible channels: direct trapping at $\mathrm{Fe}_{\mathrm{Li}}^{3+}$ sites, hopping on niobium antisites and hopping on $\mathrm{Nb}$ regular sites after conversion to the free state. Up to three regimes, each one reflecting the predominance of one of these processes, appear with different activation energies in the Arrhenius plots of the decay time. The influence of $\mathrm{Fe}$ Li and $\mathrm{Nb}_{\mathrm{Li}}$ concentrations on the transition temperatures is evidenced. For both polaron species, the length of the final hop (trapping length) is found much larger than the usual hopping length and decreases at rising temperature. This trap size effect is a natural consequence of Holstein's theory and may explain some unclear features of polaron-related light-induced phenomena, such as the temperature-dependent stretching exponent of light-induced absorption decays and the anomalous increase of the photoconductivity at high doping levels.
\end{abstract}

() 2016 Elsevier Masson SAS. All rights reserved.

\section{Introduction}

Lithium niobate $\left(\mathrm{LiNbO}_{3}\right.$, abbreviated $\left.\mathrm{LN}\right)$ is extensively studied since 40 years for its electro-optic, nonlinear and photorefractive (PR) properties, which are involved in various applications in coherent optics, telecommunications and data storage [1]. The crystal in congruent composition (CLN) is Li-deficient ([Li]/ $[\mathrm{Nb}]=0.945)$ and contains a large amount of point defects: according to the widely accepted charge compensation model [2], $1.1 \%$ of the Li sites are occupied by niobium ions (antisites $\mathrm{Nb}_{\mathrm{Li}}$ ) and $4.4 \%$ are vacant. These defects have a strong influence on the physical properties. Namely, the PR effect, defined by a change of the refractive index induced by a non-uniform illumination, is very sensitive to the $\mathrm{Li} / \mathrm{Nb}$ composition, and the role of niobium antisites in the PR effect at high light intensities has been established [3]. At low or moderate intensities, the PR effect is also sensitive to the concentrations of deep traps such as $\mathrm{Fe}^{3+}$ or $\mathrm{Cu}^{2+}[3]$. According to usual models of photo-excitation [4,5], electrons extracted from

\footnotetext{
* Corresponding author.

E-mail address: imed.mhaouech@univ-lorraine.fr (I. Mhaouech).
}

deep levels are injected into the conduction band, migrate at some distance and are finally re-trapped at other locations, leaving behind them the positive charges of ionized traps. A space charge field arises from this new charge distribution and induces a refractive index change through the electro-optic effect [6]. In irondoped $\mathrm{LN}(\mathrm{Fe}: \mathrm{LN})$, since $\mathrm{Fe}^{2+}$ are strongly photogalvanic centers in the blue-green range [4], the bulk photogalvanic current $\left(J_{p g}\right)$ is usually much larger than the diffusion current and is mainly responsible for the PR effect. Moreover, empty traps $\left(\mathrm{Fe}^{3+}\right)$ kill the photoconductivity $\left(\sigma_{p h}\right)$, leading to extremely large space charge fields $\left(E_{s c}=J_{p g} / \sigma_{p h}>10^{5} \mathrm{~V} / \mathrm{m}\right)$ and thus to large refractive index changes [6].

On another hand, it is well established that light-induced electrons in LN are trapped by Nb ions very quickly, and preferably by $\mathrm{Nb}_{\mathrm{Li}}$ antisites [7-10]. Therefore, light-induced phenomena in LN should be better described within a polaron hopping model [11] rather than usual band models [5,12]. Furthermore, since $\mathrm{Fe}_{\mathrm{Li}}$ also exhibits a large deformation change between its two charge states [13], its contribution in the dark conductivity should better be described also within the small polaron model. The absorption bands of free polarons $\left(\mathrm{Nb}_{\mathrm{Nb}}^{4+}\right)$ and bound polarons $\left(\mathrm{Nb}_{\mathrm{Li}}^{4+}\right)$ largely differ from those of bipolarons $\left(\mathrm{Nb}_{\mathrm{Li}}^{3+}\right)$ and $\mathrm{Fe}_{\mathrm{Li}}^{2+}$ [14-17]. Therefore, 
the generation of free or bound polarons by light excitation induces transient changes of the absorption spectrum, decaying as these polarons are re-trapped by impurities or recombine into bipolarons. Therefore, time-resolved light-induced absorption (LIA) gives access to polaron relaxation dynamics. In Fe:LN, the LIA signal at $785 \mathrm{~nm}$ attributed to the bound polaron $\mathrm{Nb}_{\mathrm{Li}}^{4+}$ has been found to exhibit a stretched (multi-exponential) decay on time scales ranging from nanoseconds (ns) to milliseconds [18-22]. The first comprehensive LIA study in Fe:LN under ns pulses [20] has had a major interest: it revealed the locality of the excitation process at low pulse intensity, whereas strong pulses homogenize the polaron distribution and erase the so-called site correlation effect (SCE), thus increasing drastically the decay time. Though bound and free polarons in LN are well characterized, and known to behave as small polarons in the sense of founding theories [23-27], their mobility ratio is unknown and their respective parts in lightinduced transport are still an open question; they should depend on temperature and $\mathrm{Nb}_{\mathrm{Li}}$ concentration but there is no model involving both contributions in the photoconductivity up to now. In congruent $\mathrm{LN}$, the formation of bound polarons is obviously favored owing to the large amount of $\mathrm{Nb}_{\mathrm{Li}}$ but the way they relax back to equilibrium is unclear. Several relaxation channels are possible and an important issue is to determine in which situation one or another predominates. If the deep trap concentration - say $\left[\mathrm{Fe}^{3+}\right]$ - is high, one can think that direct trapping in a single hop from $\mathrm{Nb}_{\mathrm{Li}}$ to $\mathrm{Fe}_{\mathrm{Li}}$ occurs, as assumed in Refs. [20]; in this case, the stretched decay should reflect the statistical distribution $\rho(r)$ of the $\mathrm{Nb}_{\mathrm{Li}}-\mathrm{Fe}_{\mathrm{Li}}$ distances as well as the law $\tau(r)$ of the trapping time. If the deep trap concentration is low or moderate, bound polarons can hop on the $\mathrm{Nb}_{\mathrm{Li}}$ network until they recombine into bipolarons [28] or find a deep trap; in this case the stretched decay reflects the distribution of the $\mathrm{Nb}_{\mathrm{Li}}-\mathrm{Nb}_{\mathrm{Li}}$ distances as well as the law $\tau_{h}(r)$ of the hopping time, like shown in Ref. [29]. If $\mathrm{Nb}_{\mathrm{Li}}$ also are scarce, a third possible channel for $\mathrm{Nb}_{\mathrm{Li}}^{4+}$ is to pass through the free state $\mathrm{Nb}_{\mathrm{Nb}}^{4}$, as suggested in Ref. [22].

The present paper aims to put these three processes all together in a general model and predict which one should predominate, depending on temperature and trap concentrations. The model is based on Holstein's small polaron theory [23] and Monte-Carlo (MC) simulations. We focus on the case of iron-doped material (Fe:LN) and consider that both species of electron single polarons (bound and free) can be simultaneously present. Contrarily to previous models $[29,30]$, the present one considers not only the hopping processes between equivalent sites (from $\mathrm{Nb}_{\mathrm{Nb}}$ to $\mathrm{Nb}_{\mathrm{Nb}}$ or from $\mathrm{Nb}_{\mathrm{Li}}$ to $\mathrm{Nb}_{\mathrm{Li}}$ ) and the final trapping process at a $\mathrm{Fe}_{\mathrm{Li}}$ site, but also the thermokinetic interplay between $\mathrm{Nb}_{\mathrm{Li}}^{4+}$ and $\mathrm{Nb}_{\mathrm{Nb}}^{4}$, which takes place through asymmetrical processes (trapping and detrapping) at $\mathrm{Nb}_{\mathrm{Li}}$ defects. All these processes compete in the $\mathrm{MC}$ loop and can occur a priori at any distance $r$ - not only between first neighbour sites. Their rates per unit time are supposed to depend exponentially on $r$ and 1/T. Our model thus aims to a larger scope than previous ones involving only one polaron species and no temperature dependence $[29,30]$; however, it neglects hole polarons, excitons and bipolarons, and does not simulate the photoexcitation process itself. Therefore, our MC simulations can mimic the relaxation after pulse excitation in Fe:LN materials of various $\mathrm{Fe}_{\mathrm{Li}}$ and $\mathrm{Nb}_{\mathrm{Li}}$ concentrations provided that: (i) bipolaron formation does not occur, (ii) the light-induced transfer from $\mathrm{Fe}_{\mathrm{Li}}^{2+}$ to $\mathrm{Nb}_{\mathrm{Li}}^{4+}$ is the main excitation process, (iii) the site correlation effect (SCE) addressed in Ref. [30] is excluded. The condition (i) is satisfied if the Fe content is large enough to lock the Fermi level below the bipolaron level. The condition (ii) seems more or less fulfilled under ns pulses, though electron-hole generation by two photon absorption, which prevails under fs pulses of high peak intensity [8], can also occur. Regarding the condition (iii), the pulse energy density must be large enough to kick the polarons far away from Fe traps by secondary excitations. The latter point will be re-addressed more quantitatively in Section 5.

\section{Theoretical backgrounds}

We recall here some elements of small polaron theory, essentially from early works of Holstein [23] and others [24,25]; see also $[26,27]$ and references therein. Assuming non adiabatic transfer of the carrier from site to site, the hopping rate in Holstein's molecular model [23] is given by

$w=\left(\frac{\pi}{4 k T U}\right)^{1 / 2} \frac{J^{2}}{\hbar} \exp \left(-\frac{U}{k T}\right)$

where $J$ is the transfer integral between initial and final sites, $U$ the hopping barrier, $T$ the absolute temperature and $k$ the Boltzmann constant. The formula, established in the semi-classical limit for a molecular chain, applies to a 3D crystal as well [27]. If the transport network is aperiodic or if remote transfers are considered, $J$ depends on the distance $r$ between sites. Neglecting orbital anisotropy, we assume $J(r)=I \exp (-r / 2 a)$ like in previous models $[29,31,32]$ and Eq. (1) takes the form

$w(r, T)=Z \exp \left(-\frac{r}{a}-\frac{U}{k T}\right)$,

where $a$ is an orbital parameter connected to the localization radius of the electron wave function. A trapping or de-trapping process can be considered as an asymmetrical hopping process (Fig. 1); More generally, the transfer rate between two sites $(i, j)$, equivalent or not, can be expressed by

$w_{i j}(r, T)=Z_{i j} \exp \left(-\frac{r}{a_{i j}}-\frac{U_{i j}}{k T}\right)$,

with

$Z_{i j}=\frac{g_{i j}}{2}\left(\frac{\pi}{4 k T U_{i j}}\right)^{1 / 2} \frac{I^{2}}{\hbar}$,

$U_{i j}=\frac{\left(2 E_{i}+W_{i}-W_{j}\right)^{2}}{4\left(E_{i}+E_{j}\right)}$

In these equations, the subscripts $i$ and $j$ refer to polaronic energy levels, $E_{i}$ and $E_{j}$ are polaron elastic energies, $W_{i}$ and $W_{j}$ the binding energies at zero deformation; for Eq. (5) see Refs. [25,33]. In Eq. (4), $g_{i j}$ is a coordination number, i.e. the number of symmetryequivalent sites located at equal distance from the polaron $(g=2$ in Holstein's molecular chain). The three possible values of $i$ and $j$ are $0,1,2$; they correspond respectively to $\mathrm{Nb}_{\mathrm{Nb}}, \mathrm{Nb}_{\mathrm{Li}}, \mathrm{Fe}_{\mathrm{Li}}$. For a hop between energetically equivalent sites, one has $E_{i}=E_{j}, W_{i}=W_{j}$ and Eq. (5) yields $U=E / 2$, the non-adiabatic hopping barrier.

Within usual approximations (harmonic deformation, linear coupling) the total enthalpy of a bound polaron below the reference state of Fig. 1 amounts to $E-(2 E+W) \equiv-H$, thus $H=E+W$. By definition, $H$ is larger on a trapping site (say $j$ ) than it is on a hopping site $(i<j)$, implying $E_{j}>E_{i}$ and/or $W_{j}>W_{i}$. It follows that, in most cases, Eq. (5) gives $U_{i j}<U_{i i}<U_{j i}$ for $i<j$ (see Fig. 1). Note that Eqs. (3)-(5) respect the equality $\left(U_{j i}-U_{i j}\right)=\left(H_{j}-H_{i}\right) \equiv \Delta H$ and the detailed equilibrium, $w_{j i} / w_{i j} \propto \exp -(-\Delta H / k T)$.

The validity of Eqs. (1)-(3) is restricted to non-adiabatic transfers [23] according to the criterion: 


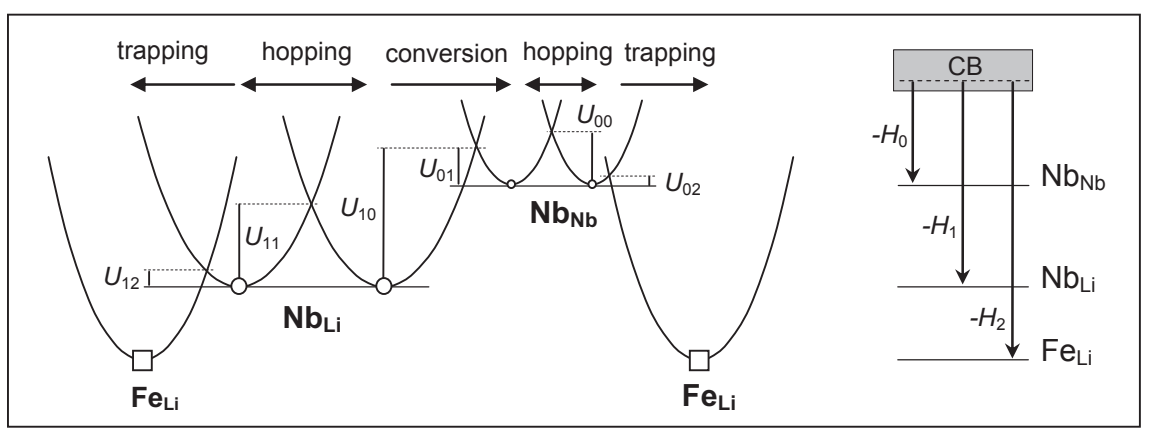

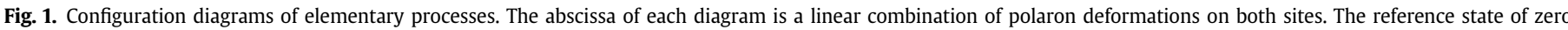
energy (dash line) is the $\mathrm{Nb}$ ground state $\left(4 d^{1}\right)$, slightly above the bottom of the $\mathrm{CB}$ in the rigid crystal.

$J^{2}<\hbar \omega_{0}(k T U)^{1 / 2}$.

In LN the phonon energy $\hbar \omega_{0}$ of the LO mode involved in polaron formation is $\sim 0.1 \mathrm{eV}$, the elastic energy $E \approx 2 U$ is estimated to $\sim 0.6 \mathrm{eV}$ for both species [17], and the transfer energy between $\mathrm{Nb}$ ions in first neighbour positions $\left(d_{0}=3.765 \AA\right)$ is $J_{0} \sim 35 \mathrm{meV}$ [34,35]. It follows that Eq. (6) is satisfied at room temperature and still far below, for free polarons hopping on the Nb sublattice, and $a$ fortiori for bound polarons hopping on the $\mathrm{Nb}_{\mathrm{Li}}$ network at larger distances ( $r \sim 3 d_{0}$ or more), with $J(r)<<J_{0}$. It should be stressed that $U$ increases with $r$ at short distance then reaches its limit value $(\sim E)$ 2 ) at about twice the polaron radius [27]. Since LN polarons are small, $U$ is virtually independent of $r$, even for free polaron hopping; experimental data also confirm that the activation energy of the dark conductivity in antisite-free material (Mg:LN, Zn:LN) is $0.25-0.29 \mathrm{eV} \approx E_{0} / 2[16,17]$.

In our study, the addressed issue is the relaxation kinetics of $\mathrm{Nb}_{\mathrm{Li}}^{4+}$ (state 1) after optical de-trapping from $\mathrm{Fe}_{\mathrm{Li}}$ (state 2). Up to six transfer processes are considered (Table 1). Thermal de-trapping $(2 \rightarrow 1)$ and hopping on the $\mathrm{Fe}_{\mathrm{Li}}$ network $(2 \leftrightarrow 2)$ are excluded because these processes - elsewhere involved in dark conduction - have no effect on the relaxation kinetics. Free polaron formation $(1 \rightarrow 0)$ (so-called conversion in the present work) and free polaron hopping $(0 \rightarrow 0)$ can be neglected or not, depending on temperature. If they are, only the first two processes of Table 1 are simulated, like in Refs. [29]; if not, all the six are considered. The values of the energy barriers $U_{i j}$ taken for the simulations (last column of Table 1) are derived from Eq. (5) with the data of Ref. [17]. The $g_{i j}$ are equal to 1 except $g_{00}=6$ (coordination of the Nb lattice). By seek of simplicity, the five parameters $a_{00}, a_{01}, a_{11}, a_{02}$ and $a_{12}$ are assumed all equal $(\equiv a)$. It is worth stressing that the difference $\delta W \equiv\left(W_{i}-\right.$ $W_{j}$ ) involved in the hopping barriers, Eq. (5), can be disturbed by the electrical potentials created by other defects of the neighbourhood; therefore, one can have $\delta W \neq 0$ even for equivalent sites, but we disregard these random potentials in the present work.

\section{Simulation methods}

First we described the simple MC method used when free polaron transport is neglected. A cubic lattice of $L^{3}$ unit cells is considered. The coordinates $\left(x_{k}, y_{k}, z_{k}\right)$ of $n_{1}$ hopping sites $\left(\mathrm{Nb}_{\mathrm{Li}}^{5+}\right)$ and $n_{2}$ trapping sites $\left(\mathrm{Fe}_{\mathrm{Li}}^{3+}\right)$ are randomized in the integer range $[1 \ldots L]$. The corresponding concentrations are $N_{1}=n_{1} / L^{3}, N_{2}=n_{2} / L^{3}$. Each hopping site is connected in the MC box to a given number of neighbour sites $\left(\mathrm{Nb}_{\mathrm{Li}}^{5+}\right.$ and $\left.\mathrm{Fe}_{\mathrm{Li}}^{3+}\right)$. Periodic boundary conditions are used, i.e., each difference $\left(x_{k}-x_{l}\right)$ is evaluated modulo $L$ for the calculations of the mutual distances $r_{k l}$ in atomic units (a.u.). Both rates $w_{11}$ (hopping) and $w_{12}$ (trapping) are assumed to decrease exponentially with $r_{k l}$ and 1/T according to Eq. (3). The MC simulation proceeds as follows:

1. The polaron is initially set at time $t=0$ on a $\mathrm{Nb}_{\mathrm{Li}}$ site $k$ chosen at random.

2. Hopping rates $w_{11}\left(r_{k l}\right)$ and trapping rates $w_{12}\left(r_{k l}\right)$ to close neighbour defects are evaluated by Eq. (3), then the sum $S$ of all of them.

3. The time $t$ is incremented by $\delta t=|\ln q| / S$, with $q$ randomized in the range $] 0 . . .1[$, so that $\delta t$ averages statistically to $1 / S$, the mean waiting time on site $k$.

4. A dice roll decides the fate of the polaron (hopping or trapping), with the dice faces sized according to the respective probabilities $w_{i j}\left(r_{k l}\right) / S$.

5. If hopping occurs, the polaron is moved to the elected site and the loop restarts from step 2; if trapping occurs, the loop ends and three quantities are memorized: the lifetime $\tau_{P}$ of the polaron, the number $n_{11}$ of hops from birth to death (excluding the final one) and the length $l_{12}$ of the final hop.

The box size $L$ is usually fixed to 21 a.u., the number $n_{1}$ of hopping sites $\left(\mathrm{Nb}_{\mathrm{Li}}^{5+}\right)$ to 100 , and the number $n_{2}$ of trapping sites $\left(\mathrm{Fe}_{\mathrm{Li}}^{3+}\right)$ to 5 or 15 ; this corresponds to Fe:CLN with a Fe doping level of $1 \times 10^{19}$ or $3 \times 10^{19} \mathrm{~cm}^{-3}$, respectively. At step 2 , it is enough to

Table 1

Polaron transfers in Fe:LiNbO 3 . The barriers $U_{i j}$ are calculated by Eq. (5) with the data of Ref. [17] $\left(E_{2}=0.70 \mathrm{eV}, W_{2}=1.22 \mathrm{eV}, E_{1}=0.58 \mathrm{eV}\right.$, $\left.W_{1}=0.53 \mathrm{eV}, E_{0}=0.545 \mathrm{eV}, W_{0}=0\right)$.

\begin{tabular}{llll}
\hline Process & Full name & \\
\hline $\mathrm{Nb}_{\mathrm{Li}}^{4+}+\mathrm{Fe}_{\mathrm{Li}}^{3+} \stackrel{w_{12}}{\longrightarrow} \mathrm{Nb}_{\mathrm{Li}}^{5+}+\mathrm{Fe}_{\mathrm{Li}}^{2+}$ & Bound polaron trapping & $U_{i j}(\mathrm{meV})$ \\
$\mathrm{Nb}_{\mathrm{Li}}^{4+}+\mathrm{Nb}_{\mathrm{Li}}^{5+} \stackrel{w_{11}}{\longrightarrow} \mathrm{Nb}_{\mathrm{Li}}^{5+}+\mathrm{Nb}_{\mathrm{Li}}^{4+}$ & Bound polaron hopping & $U_{12}=43$ \\
$\mathrm{Nb}_{\mathrm{Li}}^{4+}+\mathrm{Nb}_{\mathrm{Nb}}^{5+} \stackrel{w_{10}}{\longrightarrow} \mathrm{Nb}_{\mathrm{Li}}^{5+}+\mathrm{Nb}_{\mathrm{Nb}}^{4+}$ & Polaron conversion & $U_{10}=635$ \\
$\mathrm{Nb}_{\mathrm{Nb}}^{4+}+\mathrm{Nb}_{\mathrm{Nb}}^{5+} \stackrel{w_{00}}{\longrightarrow} \mathrm{Nb}_{\mathrm{Nb}}^{5+}+\mathrm{Nb}_{\mathrm{Nb}}^{4+}$ & Free polaron hopping & $U_{00}=272$ \\
$\mathrm{Nb}_{\mathrm{Nb}}^{4+}+\mathrm{Nb}_{\mathrm{Li}}^{5+} \stackrel{w_{01}}{\longrightarrow} \mathrm{Nb}_{\mathrm{Nb}}^{5+}+\mathrm{Nb}_{\mathrm{Li}}^{4+}$ & Free polaron bounding & $U_{01}=70$ \\
$\mathrm{Nb}_{\mathrm{Nb}}^{4+}+\mathrm{Fe}_{\mathrm{Li}}^{3+} \stackrel{w_{02}}{\longrightarrow} \mathrm{Nb}_{\mathrm{Nb}}^{5+}+\mathrm{Fe}_{\mathrm{Li}}^{2+}$ & Free polaron trapping & $U_{02}=3$ \\
\hline
\end{tabular}


consider 5 hopping sites $\left(\mathrm{Nb}_{\mathrm{Li}}^{5+}\right)$ and 5 trapping sites $\left(\mathrm{Fe}_{\mathrm{Li}}^{3+}\right)$ in the neighbourhood; we checked that remote transfers to farther sites are improbable and bring insignificant changes to the results.

Step 1 implies that the starting point has no connection to any trap; the site correlation effect (SCE) is thus disregarded. It has been reported that the SCE exists in Fe:LN at low power densities but vanishes above $[20,21]$. Our simulations do not aim to mimic this de-correlation but to account for the high power limit of LIA experiments. In this case, virtually all the $\mathrm{Fe}^{2+}$ ions are ionized, the relevant trap concentration is thus the total Fe content, and the positions of the polarons $\mathrm{Nb}_{\mathrm{Li}}^{4+}$ just after the pulse are random, just like in our simulations.

Free polaron transport is accounted for in the MC loop by adding the possibility for the bound polaron to get free. Thus, at step 2, we consider a conversion box centred on the $\mathrm{Nb}_{\mathrm{Li}}$ defect. This box assimilates the $\mathrm{Nb}$ sublattice to cubic and its size $L_{c}$ is fixed to 15 a.u., i.e. the three coordinates $(x, y, z)$ of the conversion site $\boldsymbol{r}$ can vary from -7 to +7 a.u. This procedure amounts to add $3374\left(=15^{3}-1\right)$ faces to the MC dice, each one sized proportionally to the conversion rate $w_{10}(r)$. If conversion occurs at step 4 of the primary loop, then a subroutine is launched, within which the free polaron walks away from the conversion site $(x, y, z)$ by randomly incrementing or decrementing $x, y$ or $z$, until the free polaron is trapped by a defect $\left(\mathrm{Nb}_{\mathrm{Li}}\right.$ or $\left.\mathrm{Fe}_{\mathrm{Li}}\right)$. The dice of the free polaron subroutine has got 11 faces: one of size $w_{00}$ for hopping, 5 of sizes $w_{01}\left(r_{i}\right)$ for trapping by one of the 5 closest $\mathrm{Nb}_{\mathrm{Li}}$ neighbours, 5 of sizes $w_{02}\left(r_{j}\right)$ for trapping by one of the 5 closest $\mathrm{Fe}_{\mathrm{Li}}$ neighbours. Once again, it is checked that adding more faces to this dice or enlarging the conversion box do not change the results significantly. Most often the subroutine leads to a quick re-trapping of the free polaron at the origin (the $\mathrm{Nb}_{\mathrm{Li}}$ from which conversion occurred). In this case the conversion process is said inefficient and the primary loop restarts from the previous point. If the subroutine ends on another $\mathrm{Nb}_{\mathrm{Li}}$, the conversion process is said efficient and the primary loop restarts from there. If the subroutine ends on a $\mathrm{Fe}_{\mathrm{Li}}$, the primary loop also stops. At the very end, the number $n_{10}$ of efficient conversion processes is memorized.

We test the influence of free polaron transport on bound polaron relaxation by performing some simulations with the conversion process and some others without. This is done in the same conditions at various temperatures. The loop is repeated 5000 times, renewing the site distribution each time. The survival probability $f(t)$ of the bound polaron is thus obtained with a resolution of $1 / 5000=2 \times 10^{-4}$. It can be fitted by a Kohlrausch function, $f_{K}(t)=A \exp \left[-\left(t / \tau_{K}\right)^{\beta}\right]$, as often done in experimental works $[20,22]$. Typical relaxations are shown in Fig. 2. All the memorized quantities are averaged over the 5000 runs. These averages are quoted between angular brackets in what follows.

\section{Results and discussion}

First we test the influence of the poorly-known parameter $a$. Since the transfer integral between $\mathrm{Nb}$ ions at the interatomic distance $d_{0}=3.765 \AA$ is supposed to be $J_{0}=I \exp \left(-d_{0} / 2 a\right)=35 \mathrm{meV}$ [34], we adjust the factors $I$ and $Z_{i j}$ (proportional to $I^{2}$ ) as function of $a$. We find that the bound polaron lifetime decreases by one order of magnitude with $a$ growing from 0.9 to 1.1 Å. With respect to experimental data of $\tau_{P}$ reported for Fe:CLN at $293 \mathrm{~K}$ [20,21], a fairly consistent value is $a \approx 1 \AA$. We thus keep it for our simulations throughout the paper. Note that the stretching of the decay, usually reflected by the $\beta$ exponent of the Kohlrausch fit, is also strongly influenced by $a$ [29].

Now we focus on the temperature dependences of the average number of hops $\left\langle n_{11}\right\rangle$ (Fig. 3) and the average polaron lifetime $\left\langle\tau_{P}\right\rangle$ (Fig. 4). If conversion to the free polaron state is switched off in the MC loop, two regimes are obtained. At low temperature $(T)$, $<n_{11}>$ tends to 0 : polaron relaxation thus consists in a single hop $1 \rightarrow 2$ to a Fe trap and the activation energy $E_{a}$ of $\left\langle\tau_{p}\right\rangle$ in Fig. 4a is small $(0.03 \mathrm{eV}), \approx U_{12}(0.04 \mathrm{eV})$. This is the so-called trapping (or single-hopping) regime. Above a given temperature $T_{h},<n_{11}>$ and $E_{a}$ increase (the latter tending to $\sim 0.2 \mathrm{eV}$ at high $T$ ): this is the hopping regime. One finds $E_{a}<U_{11}$, activation energy of the hopping time $(0.29 \mathrm{eV})$, because $<n_{11}>$ increases with $T$. When the conversion process is switched on, a third regime appears at high $T\left(>T_{C}\right)$, with $E_{a} \approx 0.65 \mathrm{eV} \approx U_{10}$ (conversion barrier). In this new regime, $<n_{11}>$ decreases sharply as soon as $\left\langle n_{10}\right\rangle$ (mean number of efficient conversions) increases (Fig. 3). For experimental reasons, it seems convenient to define $T_{h}$ ( $T_{c}$ respectively) as the temperature at which the average lifetime is half the extrapolated value obtained with the Arrhenius slope of the low (respectively high) temperature regime. Note that $T_{h}$ increases with the Fe concentration (Fig. 4) whereas $T_{c}$ increases with the antisite concentration (see inset of Fig. 5). With the data of Ref. [17], we find that $T_{c}$ goes down from $\sim 600 \mathrm{~K}$ to $\sim 400 \mathrm{~K}$ when the $\mathrm{Nb}_{\mathrm{Li}}$ concentration decreases from $3 \times 10^{20} \mathrm{~cm}^{-3}$ to $10^{20} \mathrm{~cm}^{-3}$. In the latter case - and a fortiori for weaker antisite concentrations - the hopping regime with activation energy $\sim 0.2 \mathrm{eV}$ becomes hardly visible on the Arrhenius plot of the polaron lifetime (Fig. 5). Our results thus explain why very different values, ranging from 0.15 to $0.62 \mathrm{eV}$, have been reported for the activation energy of the LIA decay time in Fe:LN for various $\mathrm{Nb} / \mathrm{Li}$ ratios and Fe concentrations [20,21]. It is worth stressing that in real crystals with some disordered potentials, the activation energies would be slightly different than those obtained here [36], and probably the transition temperatures as well.

The $T$-dependence of the $\beta$ exponent is another important finding. Fig. 4b shows a drastic increase of $\beta$ through the gradual transitions from trapping to hopping then from hopping to conversion. This tendency, experimentally evidenced in Refs. [20], has not been fully explained. The statistical disorder of the hopping barriers was proposed as a possible origin of the phenomenon [29]. Our results suggest an alternative explanation. In the trapping regime, $\beta$ is a constant which depends only on the statistical distribution of the distances to the nearest trap. Note that this low- $T$ limit of $\beta$ is only indicative because the KWW fit is usually coarse in the trapping regime. Then, in the hopping regime, $\beta(T)$ increases, even though the conversion process is not activated (open symbols with dash lines in Fig. 4b), because the lengthening of the random walks (evidenced in Fig. 3a) tends to re-equilibrate the lifetimes. Without the conversion process, the limit of $\beta$ at $T \rightarrow \infty$ would be the value reported in Ref. [29] and determined only by the dimensionless parameters $N_{1} a^{3}$ and $N_{2} / N_{1}$. When the conversion process is switched on (solid symbols with solid lines in Fig. 4b), $\beta(T)$ increases much more steeply and its high- $T$ limit becomes very large $(\sim 1)$. This is because the diffusion is boosted by free polaron transport, making the $\mathrm{Nb}_{\mathrm{Li}}^{4+}$ population decay less sensitive to the randomness of the $\mathrm{Nb}_{\mathrm{Li}}$ network; namely, conversion to the free state helps the bound polaron to escape from dead-end paths ending on isolated antisites. This is also the reason why $\left\langle n_{11}>\right.$ finds a maximum then decreases sharply at rising $T$ (Fig. 3a). Note that $\beta(T)$ in Fig. 4b shows the same tendency as in Ref. [20] but does not fit exactly the experimental plot reported therein; the discrepancy may come from the fact that we did not consider random potentials in our simulations, or from our assumption on the orbital parameters, $a_{i j} \equiv a=1 \AA$. Though acceptable in a first step, this assumption could be refined in the future: since the $J_{i j}$ transfer integrals link not only $\mathrm{Nb}$ orbitals together but also Fe orbitals to $\mathrm{Nb}$ orbitals, one rather expects $a_{00} \approx a_{01} \approx a_{11} \neq a_{02} \approx a_{12}$. However the goal of the paper is not to identify accurately these $a_{i j}$ but to show how the relaxation kinetics changes when several processes compete.

It should be stressed that the onset of polaron conversion to free 


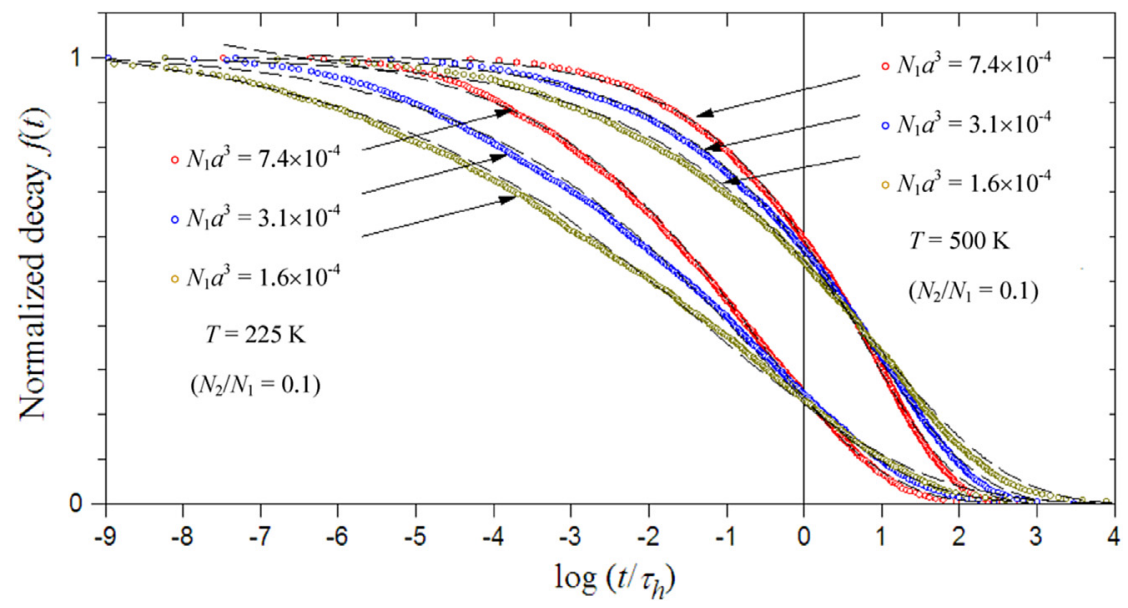

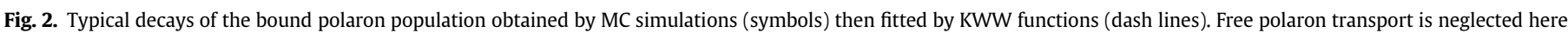
The time unit $\tau_{h}$ is the hopping time at the distance $l_{h}=\left(2 \pi N_{1}\right)^{-1 / 3}$ (most probable distance between neighbour antisites).

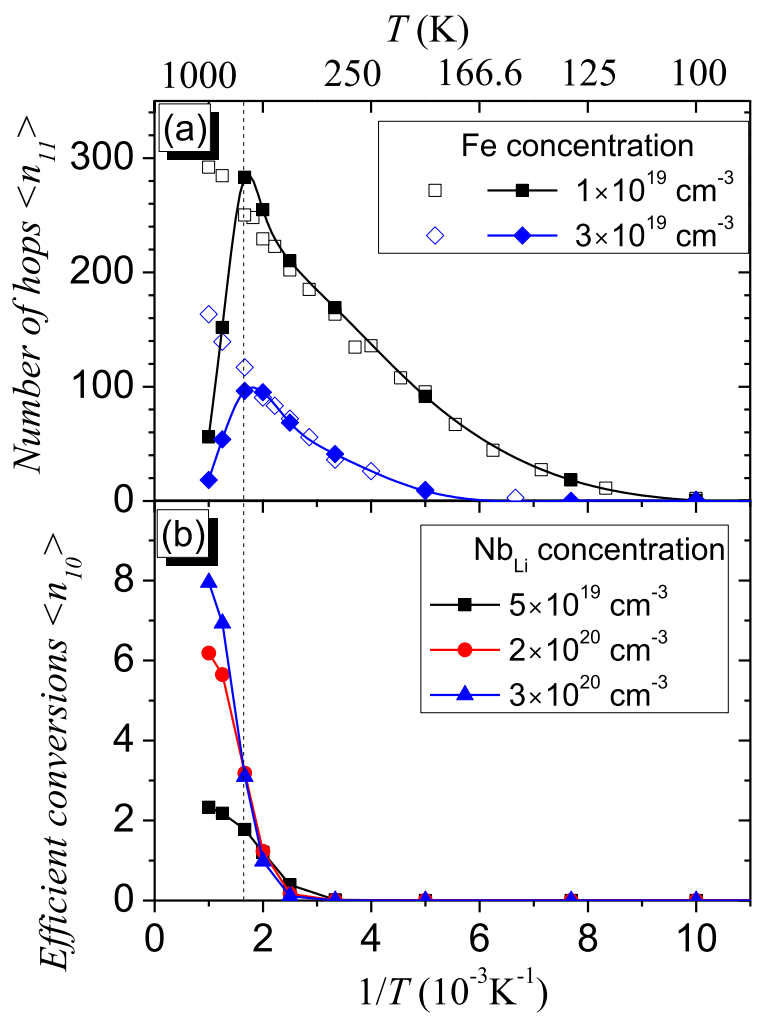

Fig. 3. Mean numbers of hops (a) and conversions (b) of the bound polaron on its random walk until trapping. Open symbols in Fig. 3a are the results obtained with the conversion to free polaron switched off in the MC loop.

state (Figs. 4a and 5) does not mean that most of bound polarons turn into free ones. The lifetime of the free polaron is always very short compared to the waiting time on the antisite before conversion. Correlatively, the equilibrium population ratio of free polarons versus bound polarons, $N_{F} / N_{P} \approx\left(N_{0} / N_{1}\right) \exp -(-\Delta H / k T)$, is always very small $\left(<2 \times 10^{-3}\right.$ at $600 \mathrm{~K}$ with the data of Table 1$)$. Obviously, free polarons boost the transport above $T_{c}$, even though they are very few, because they are much more mobile than bound polarons.

It is also worth commenting on the $T$-dependence of the mean trapping lengths $\left\langle l_{01}\right\rangle,\left\langle l_{02}\right\rangle,\left\langle l_{12}\right\rangle$ (Fig. 6). The latter (trapping length of the bound polaron by $\mathrm{Fe}_{\mathrm{Li}^{+}}^{3+}$ ) is recorded during the MC

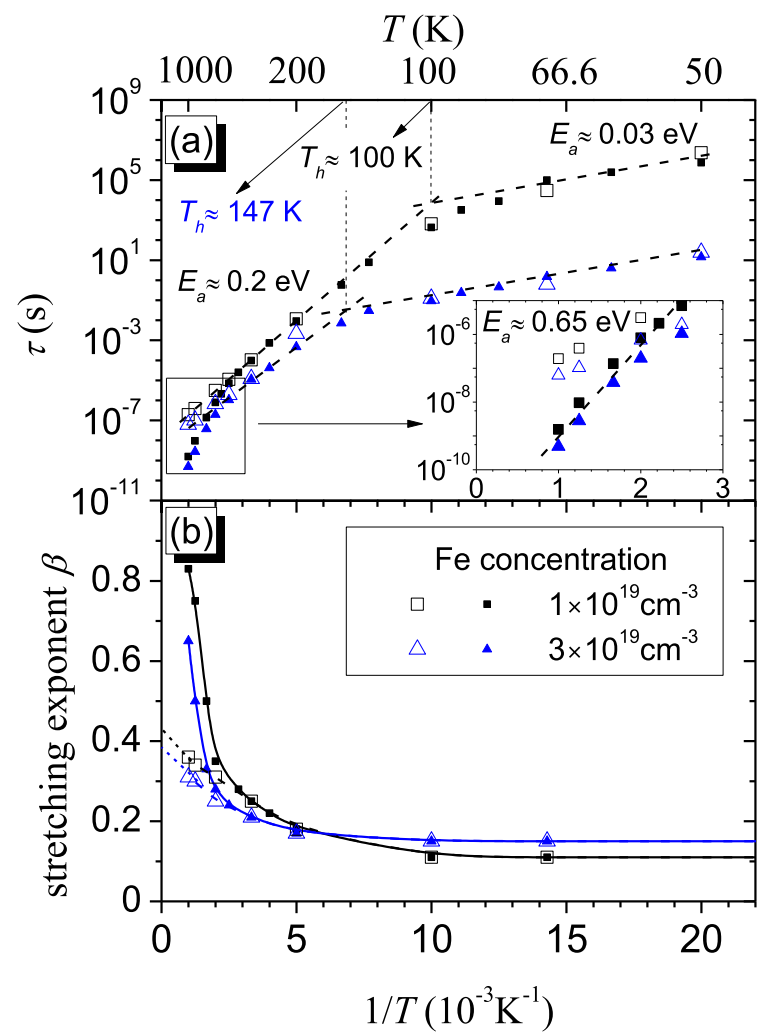

Fig. 4. Bound polaron lifetime (a) and stretching exponent (b), with and without the conversion process (solid and open symbols respectively). The KWW fit is made on the 5000 points of each MC decay. The $\mathrm{Nb}_{\mathrm{Li}}$ concentration is $2 \times 10^{20} \mathrm{~cm}^{-3}$ (CLN case).

loop described in the first part of section 3 (conversion off). The former two (trapping lengths of the free polaron by $\mathrm{Nb}_{\mathrm{Li}}^{5+}$ and $\mathrm{Fe}_{\mathrm{Li}}^{3+}$ respectively) are obtained after 5000 runs of a quick loop simulating the walk of a free polaron among one trap species only (either $\mathrm{Nb}_{\mathrm{Li}}^{5+}$ or $\mathrm{Fe}_{\mathrm{Li}}^{3+}$ ). All these trapping lengths increase almost linearly with $T^{-1}$ at high $T$ then reach a limit at low $T$. Therefore, it is clear that polaron traps have an effective spatial extension, decreasing at rising $T$. This important result can be interpreted by estimating at which distance $d_{i j}$ a polaron on a site $i$ gets equal chances to be trapped by $j$ or to escape on another equivalent site $i^{\prime}$ located at a typical hopping distance $d_{i}$, proportional to $N_{i}^{-1 / 3}$. At the 


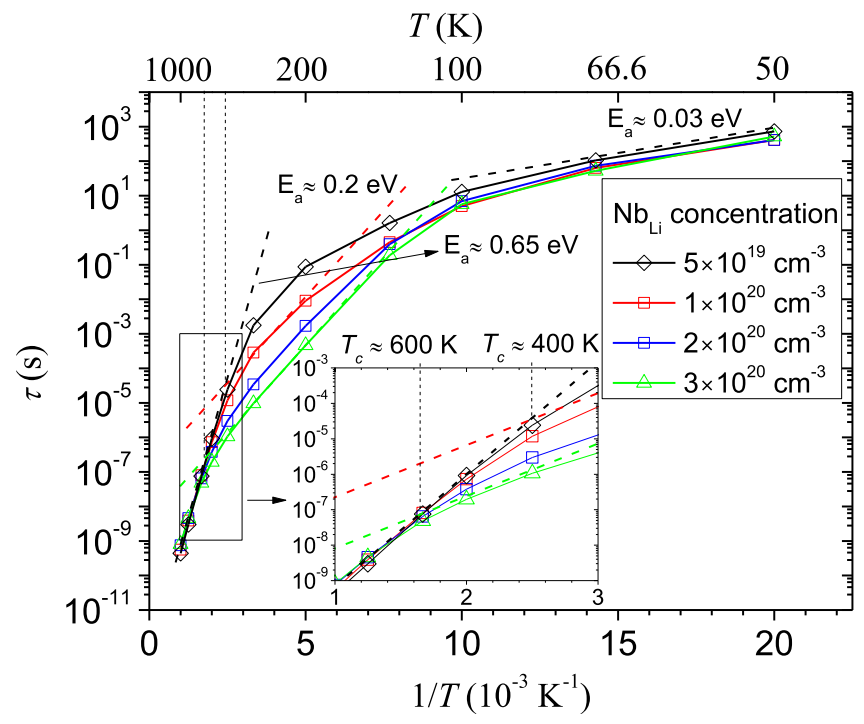

Fig. 5. Bound polaron lifetime in Fe:LN of various Li contents ranging from nearly stoichiometric to sub-congruent. The Fe concentration is fixed here to $2 \times 10^{19} \mathrm{~cm}^{-3}$.

distance $d_{i j}$, the trapping rate to an isolated trap would be approximately equal to the hopping rate $w_{i i}\left(d_{i}\right)$. More precisely, the site $i^{\prime}$ has got nearly equal chances to be at a smaller or larger distance $d_{i^{\prime} j}$ from the trap, and most probably the polaron will escape only in the latter case but be trapped in the former case. It follows that $w_{i j}\left(d_{i j}\right) \approx w_{i i}\left(d_{i}\right) / 2$, from which one readily finds:

$d_{i j}(T) \approx d_{i}+a \ln \left(\frac{2 Z_{i j}}{Z_{i i}}\right)+a \frac{U_{i i}-U_{i j}}{k T}$

In the second member of Eq. (7), the first term $d_{i}$ is the hopping distance; for free polarons, it equals the $\mathrm{Nb}-\mathrm{Nb}$ distance $\left(d_{0}=3.765 \AA\right)$, and for bound polarons the mean distance to the nearest $\mathrm{Nb}_{\mathrm{Li}}$ site $\left(d_{1}=9.5 \AA\right.$ in $\left.\mathrm{CLN}\right)$. The second term is ordinary small but the third one $(>0)$ becomes large at low $T$. The distance $d_{i j}$ given by Eq. (7) can be seen as the radius of a trapping sphere surrounding each trap and shrinking at rising $T$. This so-called trap size effect (TSE) is shown in Fig. 8. Obviously, the mean trapping length $\left\langle l_{i j}>\right.$ estimated by MC simulations can be close to the expected value $d_{i j}$ only for isolated traps (far away from each other); therefore $d_{i j}(T)$ is an asymptotic limit for $<l_{i j}>$ at high $T$, as can be seen in Fig. 6 for $\left\langle l_{01}>\right.$ and $\left\langle l_{02}>\right.$. The case of $\left\langle l_{12}>\right.$ is slightly different. If the conversion process is switched off in MC simulations, the Fe trap size actually shrinks like $a\left(U_{11}-U_{12}\right) / k T$ and tends typically to the mean hopping distance $d_{1}$ for $T \rightarrow \infty$, as shown by a linear extrapolation in Fig. 6; however, conversion settles at a finite $T_{c}$, above which the transport cannot be described in terms of bound polaron hopping anymore, since free polaron transport becomes predominant. When conversion is efficient (thus above $T_{c}$ ) the trapping length $\left\langle l_{12}>\right.$ shrinks more quickly than below $T_{C}$, typically like $a\left(U_{10}-U_{12}\right) / k T$, as shown in Fig. 7.

For free polarons, according to our MC simulations, $\mathrm{Fe}^{3+}$ has a trapping radius bigger than that of $\mathrm{Nb}_{\mathrm{Li}}^{5+}$. This is a consequence of a smaller trapping barrier, $U_{02}<U_{01}$ (Table 1). With $a=1 \AA$ and the data of Ref. [17], Eq. (7) gives $d_{01} \approx 11 \AA$ And $d_{02} \approx 15 \AA$ at $293 \mathrm{~K}$. The efficient conversion length $\left\langle l_{10}>\right.$, elsewhere estimated through the full MC loop, is found much larger than $d_{0}$ (interatomic distance), except at $T \rightarrow \infty$. It means that electronic transfers from $\mathrm{Nb}_{\mathrm{Li}}$ to a first neighbour $\mathrm{Nb}$ site are inefficient because the newborn free polaron is immediately re-trapped by the antisite. Only remote conversions are efficient, and the average distance $<l_{10}>$ at which

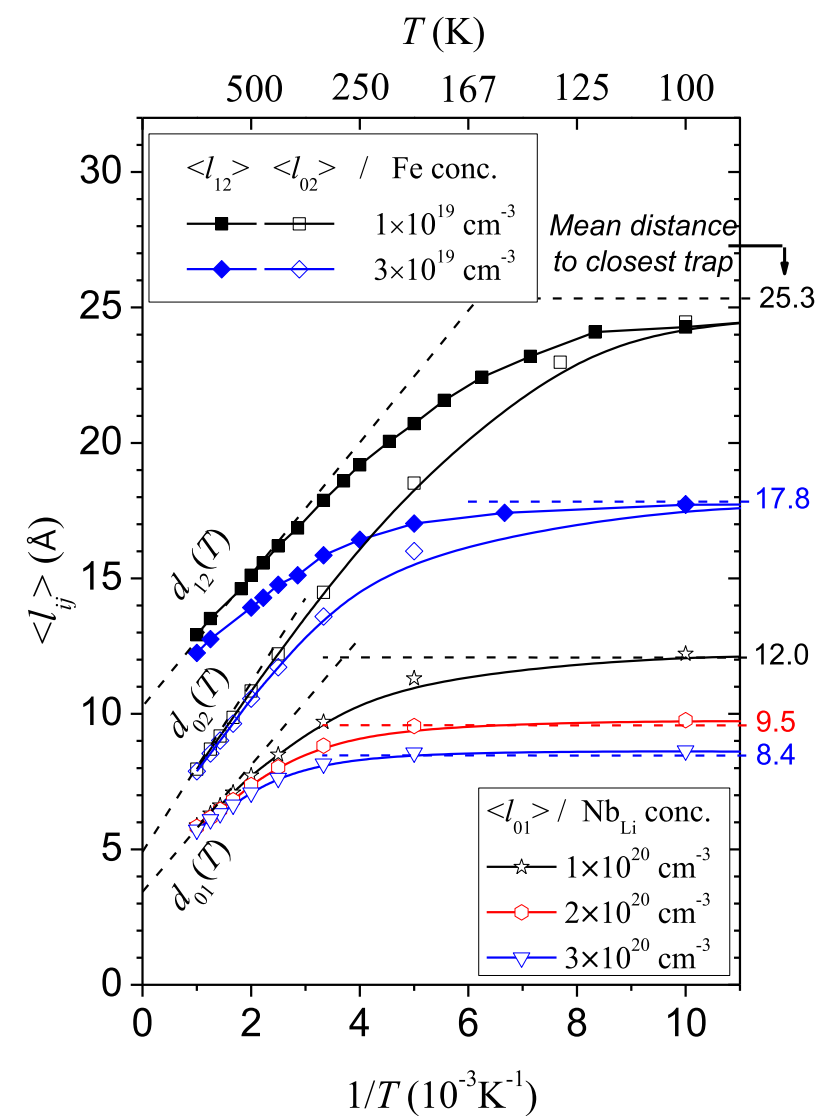

Fig. 6. Mean trapping lengths $<l_{i j}>$ for various concentrations of $\mathrm{Fe}^{3+} \operatorname{traps}(j=2)$ and $\mathrm{Nb}_{\mathrm{Li}}^{5+}$ traps $(j=1)$. Subscript $i$ refers to the carrier ( 0 for free polaron, 1 for bound polaron). Conversion is switched-off to determine $\left\langle l_{12}\right\rangle$. The asymptotic limits (dash lines) are the trapping distances $d_{i j}(T)$ given by Eq. (7) for high temperatures and the mean distances $d_{j}$ to the nearest trap in the cubic structure for low temperatures.

they occur is found equal to $<l_{01}>$, as expected by virtue of the detailed equilibrium.

The increase of $\left\langle l_{i j}>\right.$ with $T^{-1}$ is no longer linear when the trapping regime settles, that is, when the trapping spheres begin to overlap (Fig. 8c). In this regime $\left\langle l_{i j}>\right.$ tends to a low- $T$ limit equal to the mean distance to the nearest trap, $d_{j} \approx\left(2 \pi N_{j}\right)^{-1 / 3}$, as it can be checked in Fig. 6.

\section{Conclusion}

A first important result of this work is that the lifetime of bound polarons among deep traps cannot follow a single Arrhenius law. As a rule, when the simulation takes account of all possible processes on the basis of Holstein's model, several regimes are observed. Direct trapping predominates at low temperature, then a multihopping regime settles upon heating. The activation energies of these two regimes are very different and slightly lower than the corresponding barriers. At high temperature, a third regime appears, within which polarons diffuse in the free state more efficiently than by hopping on shallow traps. This so-called conversion regime settles at a lower temperature if the shallow trap concentration is smaller. Fig. 9 schematically recapitulates the three regimes versus temperature and shows how their transitions are influenced by the trap concentrations. When $T_{c}$ and $T_{h}$ are not very different, like in the case of iron-doped nearly stoichiometric LN (Fe:NSLN), the hopping regime can virtually disappear and the activation energy of the bound polaron lifetime is expected much 


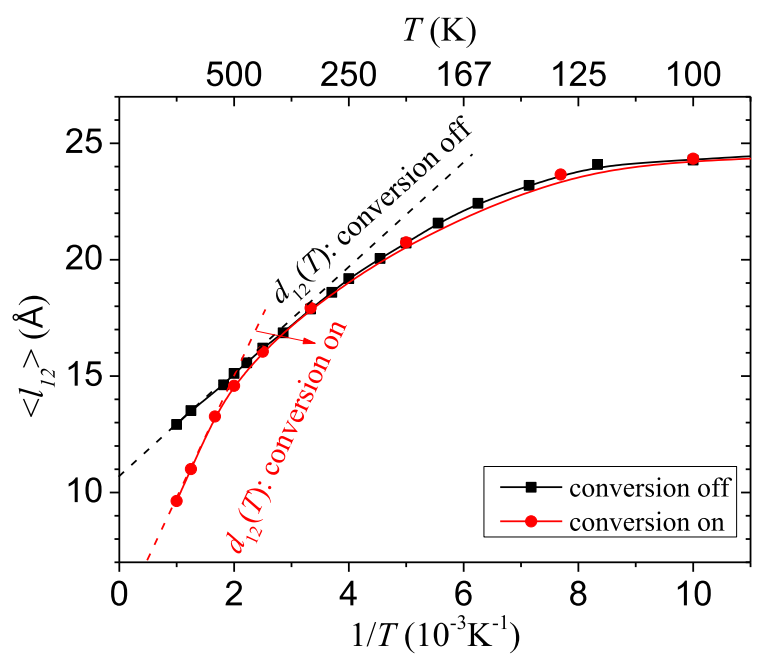

Fig. 7. Mean trapping lengths $<l_{12}>$ with and without the conversion of bound polaron to free polaron. The Fe concentration is fixed to $1 \times 10^{19} \mathrm{~cm}^{-3}$.

higher than in the CLN case. This was clearly evidenced by LIA experiments, e.g. $E_{a} \sim 0.62 \mathrm{eV}$ in $\mathrm{Fe}: \operatorname{NSLN}(49.6 \% \mathrm{~mol} \mathrm{Li})$ instead of $E_{a}<0.3 \mathrm{eV}$ in Fe:CLN [20].

This work also brings a new explanation for the $T$-dependence of the stretching exponent. The previous interpretation based on disorder [29] would be probably valid if the relaxing particles were electrons with no (or weak) polaronic character, hopping across random potentials $V$ of large standard deviation $\delta V$, like e.g. electrons hopping on shallow traps in silicon at low temperature [31]. In this case, there would be no difference between hopping and trapping barriers, and the trapping sites would have no sizeable extension in the network, like assumed in Ref. [29]. The case of small polarons in LN is different: the strong electron-phonon coupling induces large differences between hopping and trapping barriers, $\left(U_{i i}-U_{i j}\right) \equiv \delta U_{i j}>>\delta V$. A trap size effect (TSE), that is, a $T$ dependent trapping radius, is thus predicted according to Eq. (7). This TSE, clearly evidenced in Figs. 6-7 and schematically illustrated in Fig. 8, appears as a direct consequence of $\delta U_{i j}$ and is thus a general property of polaron traps. As soon as $\delta U_{i j}>\delta V$, the TSE is probably the major effect (versus disorder) and should play the leading role in $T$-dependent phenomena related to polaron transport.

Furthermore, the TSE helps to describe clearly the SCE. The decorrelation threshold reported in Ref. [21] $\left(\sim 160 \mathrm{MW} / \mathrm{cm}^{2}\right)$ corresponds to a photonic density of $10^{18}$ photons $/ \mathrm{cm}^{2}$ per pulse, $\sim 4 / S_{P}$ (with $S_{P}$ the absorption cross section of $\mathrm{Nb}_{\mathrm{Li}}^{4+}$ ). It means that most of the bound polarons are kicked away from their starting Fe site when each of them has been hit by 4 photons (in average) during the pulse. For lower photonic densities, some polarons stay inside the trapping sphere of their Fe site and are most often re-trapped in a single hop, even from distances as large as $d_{12}(\sim 20 \AA$ in CLN at $293 \mathrm{~K}$ ), thus much farther than the nearest $\mathrm{Nb}_{\mathrm{Li}}$ neighbour. We may thus predict that in the SCE regime, the activation energy of the polaron lifetime should go down to $U_{12} \sim 0.04 \mathrm{eV}$, like in the trapping regime.

The scope of the TSE spreads far beyond light-induced absorption and may also explain several unclear features of other lightinduced effects versus temperature, antisite concentration and deep trap concentration. Namely, the big trapping radii estimated for $\mathrm{Fe}^{3+}$ in this work could be responsible for the weak photoconductivity of Fe:CLN, for its non-Arrhenius dependence and for its anomalous behaviour at high doping levels [37]. The latter feature can be explained by the overlapping of the trapping spheres, which facilitates light-induced transfers from $\mathrm{Fe}^{2+}$ to $\mathrm{Fe}^{3+}$ (Fig. 8c) instead

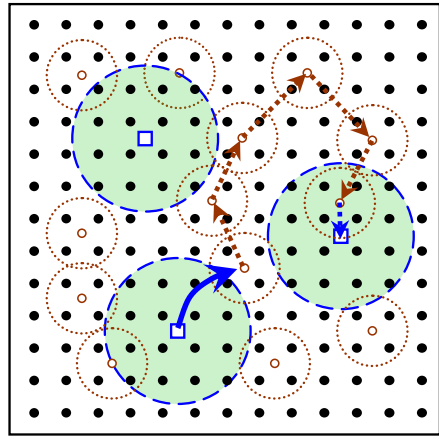

(a) $500 \mathrm{~K}$

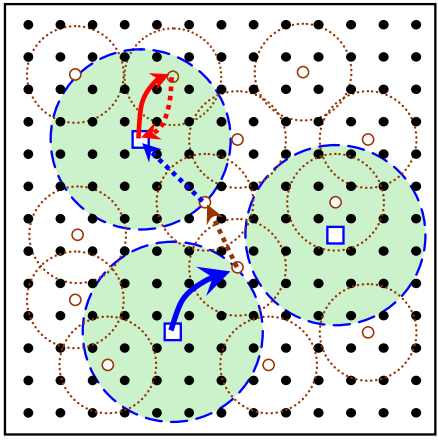

(b) $300 \mathrm{~K}$

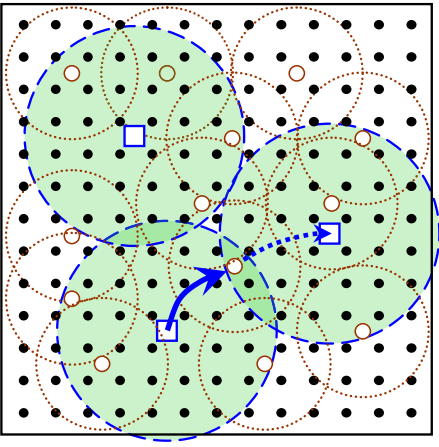

(c) $200 \mathrm{~K}$

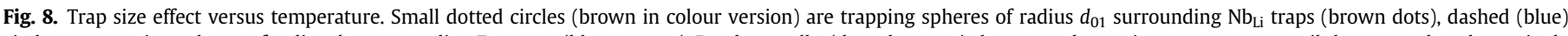

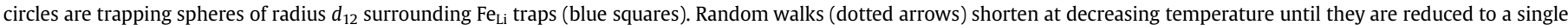

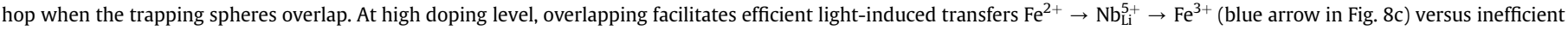
ones (red arrow in Fig. 8b). (For interpretation of the references to colour in this figure legend, the reader is referred to the web version of this article.)

\begin{tabular}{ccc} 
Low $\stackrel{\left[\mathrm{Fe}^{3+}\right]}{\longleftarrow}$ High & Low $\stackrel{\left[\mathrm{Nb}_{\mathrm{Li}}^{5+}\right]}{\stackrel{T_{C}}{\longrightarrow}}$ High \\
\hline $\begin{array}{c}\text { Direct trapping to } \\
\text { deep levels }\end{array}$ & $\begin{array}{c}\text { Multi-hopping } \\
\text { regime }\end{array}$ & $\begin{array}{c}\text { Polaron conversion to } \\
\text { free polaron }\end{array}$ \\
$E_{a} \sim 0.03 \mathrm{eV}$ & $E_{a} \sim 0.2 \mathrm{eV}$ & $E_{a} \sim 0.65 \mathrm{eV}$
\end{tabular}

Fig. 9. Diagram showing the sequence of polaron decay regimes at rising temperature. 
of direct re-trapping of the polaron by the Fe ion (Fig. 8b).

However, the estimates given in this paper must be refined. Some polaron data used to calculate the barriers $U_{i j}$ are still uncertain (see Appendix) and the orbital parameters $a_{i j}$ are also poorly known. Detailed comparisons of MC predictions to experimental LIA data should permit one to extract some missing parameters. Unfortunately, LIA data versus temperature in Fe:LN are still insufficient and limited to narrow T-ranges. Namely, new LIA experiments in Fe:CLN below $200 \mathrm{~K}$ are necessary to check whether the activation energy of the polaron lifetime $\left\langle\tau_{P}\right\rangle$ becomes small and the stretching exponent $\beta$ tends to a low limit, possibly giving access to $a_{12}$. On another hand, in the hopping regime, the $T$-dependences of both $\left\langle\tau_{P}\right\rangle$ and $\beta$ should give access to refined values of both $U_{11}\left(\approx E_{1} / 2\right)$ and $a_{11}$, whereas the activation energy of $\left\langle\tau_{P}\right\rangle$ in NSLN at high temperature should permit one to refine $U_{10}$, the conversion barrier. The model presented in this paper and the socalled trap size effect evidenced by the simulations can serve as a solid basis to better explain the light-induced properties of $\mathrm{LiNbO}_{3}$, not only light-induced absorption at micro or nanosecond scale, but also photoconduction and photorefractive effects, under pulse excitation or constant wave as well.

\section{Acknowledgements}

The authors are grateful to Region Lorraine for financial support of their research works and would like to thank Prof. G. Montemezzani (University of Lorraine) and Dr. M. Bazzan (University of Padova) for useful discussions and critical reading.

\section{Appendix. Polaron energies}

The elastic energy $E$ of a small polaron is experimentally accessible, either from the activation energy of the electrical conductivity ( $U=E / 2$ in non adiabatic regime) or from spectroscopic data [17]. For a bound polaron, there is a binding energy $W$, which is the additional potential energy of the carrier localized on the defective site at null deformation. Within the simple polaron model assuming harmonic deformation and linear coupling, that is, the elastic energy $E$ proportional to $Q^{2}$ (square of the deformation) and the electronic energy shift $E_{\text {elec }}$ (gained in the deformation) proportional to $Q$ one finds $E_{\text {elec }}=-2 E$ in the equilibrium state, the optical absorption band peaks at $M=(W+2 E)$ and its width is proportional to $\left(E \hbar \omega_{0}\right)^{1 / 2}$. It follows that both $E$ and $W$ can be deduced from the polaron absorption spectrum, if the phonon energy $\hbar \omega_{0}$ of the LO mode involved in the deformation is known. Assuming $\hbar \omega_{0}=0.10 \mathrm{eV}$ (typical value for oxides), Schirmer et al. [17] estimated in this way the polaron energies in $\mathrm{LiNbO}_{3}$ :

$$
\begin{aligned}
& \mathrm{Nb}_{\mathrm{Nb}}^{4+}: M_{0}=1.09 \mathrm{eV}, E_{0}=0.54 \mathrm{eV}\left(\text { since } W_{0}=0\right) \\
& \mathrm{Nb}_{\mathrm{Li}}^{4+}: M_{1}=1.69 \mathrm{eV}, E_{1}=0.58 \mathrm{eV}, W_{1}=0.53 \mathrm{eV} \\
& \mathrm{Fe}_{\mathrm{Li}}^{2+}: M_{2}=2.62 \mathrm{eV}, E_{2}=0.70 \mathrm{eV}, W_{2}=1.22 \mathrm{eV}
\end{aligned}
$$

Putting these data in Eq. (5), one finds the values of Table 1 for the barriers $U_{i j}$ used in the present work. The value $\hbar \omega_{0}=0.1 \mathrm{eV}$ is consistent with the breathing mode of the $\mathrm{O}_{6}$ octahedron around $\mathrm{Nb}$ ions, and $E_{0} \approx 0.5 \mathrm{eV}$ is redundantly confirmed by experimental data; see Ref. [17] and references therein. However, the phonon energy $\hbar \omega_{1}$ of $\mathrm{Nb}_{\mathrm{Li}}^{4+}$ may be lower than $0.1 \mathrm{eV}$ because the distortion around the antisite probably involves also the stretching of the cationic bond $\mathrm{Nb}-\mathrm{Nb}$ along the polar axis [38], which is much softer than the $\mathrm{O}_{6}$ breathing mode. Revising $\hbar \omega_{1}$ implies to revise both $E_{1}$ and $W_{1}$, as well as the difference $\delta U_{12}=U_{11}-U_{12}$ ), which determines the effective trap size of $\mathrm{Fe}^{3+}$ and thus the extrinsic lightinduced properties of Fe:LN. Moreover, $\hbar \omega_{1}$ influences also the conversion barrier $U_{10}$, thus the conversion temperature and the intrinsic properties of Li-deficient LN. In the extreme case, if one sets $W_{1} \sim 0$ (virtually no attractive potential on $\mathrm{Nb}_{\mathrm{Li}}$ ), one would get $\hbar \omega_{1}=70 \mathrm{meV}, U_{11}=0.41 \mathrm{eV}, U_{01}=0.2 \mathrm{eV}$ (instead of $0.07 \mathrm{eV}$ ), $\delta U_{12}=0.37 \mathrm{eV}$ (instead of $0.25 \mathrm{eV}$ ). On the other hand, ab initio calculations [38] suggest that the linear approximation $\left(E_{\text {elec }} \propto-Q\right)$ is rough for $\mathrm{Nb}_{\mathrm{Li}}^{4+}$. The phonon energy of the deep polaron $\mathrm{Fe}_{\mathrm{Li}}^{2+}$ also may differ more or less from $0.1 \mathrm{eV}$ and affect both $U_{12}$ and $U_{02}$. It is thus essential to investigate more accurately the structures of bound polarons in $\mathrm{LN}$ and refine their energies.

\section{References}

[1] L. Arizmendi, Photonic applications of lithium niobate crystals, Phys. Status Solidi A 201 (2004) 253-283, http://dx.doi.org/10.1002/pssa.200303911.

[2] P. Lerner, C. Legras, J.P. Dumas, Stoechiométrie des monocristaux de métaniobate de lithium, J. Cryst. Growth. (3-4) (1968) 231-235, http:// dx.doi.org/10.1016/0022-0248(68)90139-5.

[3] T. Volk, M. Wöhlecke, Lithium Niobate: Defects, Photorefraction and Ferroelectric Switching, Springer Science \& Business Media, Berlin Heidelberg, 2008.

[4] A.M. Glass, D. von der Linde, T.J. Negran, High-voltage bulk photovoltaic effect and the photorefractive process in LiNbO3, Appl. Phys. Lett. 25 (1974) 233-235, http://dx.doi.org/10.1063/1.1655453.

[5] N.V. Kukhtarev, V.B. Markov, S.G. Odulov, M.S. Soskin, V.L. Vinetskii, Holographic storage in electrooptic crystals. i. steady state, Ferroelectrics 22 (1978) 949-960, http://dx.doi.org/10.1080/00150197908239450.

[6] E. Krätzig, O.F. Schirmer, Photorefractive centers in electro-optic crystals, in: P. Günter, J.-P. Huignard (Eds.), Photorefractive Materials and Their Applications I, Springer-Verlag, Berlin, 1988, pp. 131-166.

[7] Y. Qiu, K.B. Ucer, R.T. Williams, Formation time of a small electron polaron in $\mathrm{LiNbO}_{3}$ : measurements and interpretation, Phys. Status Solidi C 2 (2005) 232-235, http://dx.doi.org/10.1002/pssc.200460153.

[8] O. Beyer, D. Maxein, T. Woike, K. Buse, Generation of small bound polarons in lithium niobate crystals on the subpicosecond time scale, Appl. Phys. B 83 (2006) 527-530, http://dx.doi.org/10.1007/s00340-006-2227-9.

[9] P. Reckenthaeler, D. Maxein, T. Woike, K. Buse, B. Sturman, Separation of optical Kerr and free-carrier nonlinear responses with femtosecond light pulses in $\mathrm{LiNbO}_{3}$ crystals, Phys. Rev. B 76 (2007) 195117, http://dx.doi.org/ 10.1103/PhysRevB.76.195117.

[10] S. Sasamoto, J. Hirohashi, S. Ashihara, Polaron dynamics in lithium niobate upon femtosecond pulse irradiation: influence of magnesium doping and stoichiometry control, J. Appl. Phys. 105 (2009) 83102, http://dx.doi.org/ 10.1063/1.3095763.

[11] B. Sturman, M. Carrascosa, F. Agullo-Lopez, Light-induced charge transport in $\mathrm{LiNbO}_{3}$ crystals, Phys. Rev. B 78 (2008) 245114, http://dx.doi.org/10.1103/ PhysRevB.78.245114.

[12] F. Jermann, J. Otten, Light-induced charge transport in $\mathrm{LiNbO}_{3}: \mathrm{Fe}$ at high light intensities, J. Opt. Soc. Am. B 10 (1993) 2085, http://dx.doi.org/10.1364/ JOSAB.10.002085.

[13] A. Sanson, A. Zaltron, N. Argiolas, C. Sada, M. Bazzan, W.G. Schmidt, S. Sanna, Polaronic deformation at the $\mathrm{Fe}^{2+/ 3+}$ impurity site in $\mathrm{Fe}: \mathrm{LiNbO}_{3}$ crystals, Phys. Rev. B 91 (2015) 94109, http://dx.doi.org/10.1103/PhysRevB.91.094109.

[14] J. Koppitz, O.F. Schirmer, A.I. Kuznetsov, Thermal dissociation of bipolarons in reduced undoped $\mathrm{LiNbO}_{3}$, EPL Europhys. Lett. 4 (1987) 1055, http://dx.doi.org/ 10.1209/0295-5075/4/9/017

[15] K.L. Sweeney, L.E. Halliburton, Oxygen vacancies in lithium niobate, Appl. Phys. Lett. 43 (1983) 336-338, http://dx.doi.org/10.1063/1.94347.

[16] B. Faust, H. Müller, O.F. Schirmer, Free small polarons in $\mathrm{LiNbO}_{3}$, Ferroelectrics 153 (1994) 297-302, http://dx.doi.org/10.1080/00150199408016583.

[17] O.F. Schirmer, M. Imlau, C. Merschjann, B. Schoke, Electron small polarons and bipolarons in $\mathrm{LiNbO}_{3}$, J. Phys. Condens. Matter 21 (2009) 123201, http:// dx.doi.org/10.1088/0953-8984/21/12/123201.

[18] M. Simon, F. Jermann, E. Krätzig, Light-induced absorption changes in irondoped $\mathrm{LiNbO}_{3}$, Opt. Mater. 3 (1994) 243-250, http://dx.doi.org/10.1016/ 0925-3467(94)90036-1.

[19] F. Jermann, M. Simon, R. Bower, E. Kratzig, O.F. Schirmer, Light-induced absorption changes in reduced lithium niobate, Ferroelectrics 165 (1995) 319-327, http://dx.doi.org/10.1080/00150199508228312.

[20] D. Berben, K. Buse, S. Wevering, P. Herth, M. Imlau, T. Woike, Lifetime of small polarons in iron-doped lithium-niobate crystals, J. Appl. Phys. 87 (2000) 1034-1041, http://dx.doi.org/10.1063/1.371976.

[21] P. Herth, D. Schaniel, T. Woike, T. Granzow, M. Imlau, E. Krätzig, Polarons generated by laser pulses in doped $\mathrm{LiNbO}_{3}$, Phys. Rev. B 71 (2005) 125128, http://dx.doi.org/10.1103/PhysRevB.71.125128.

[22] C. Merschjann, D. Berben, M. Imlau, M. Wöhlecke, Evidence for two-path recombination of photoinduced small polarons in reduced $\mathrm{LiNbO}_{3}$, Phys. Rev. Lett. $96 \quad$ (2006) 186404, http://dx.doi.org/10.1103/ PhysRevLett.96.186404.

[23] T. Holstein, Studies of polaron motion, Ann. Phys. 8 (1959) 343-389, http:// dx.doi.org/10.1016/0003-4916(59)90003-X.

[24] I.G. Lang, Y.A. Firsov, Kinetic theory of semiconductors with low mobility, Sov. 
Phys. JETP 16 (1963).

[25] I.G. Austin, N.F. Mott, Polarons in crystalline and non-crystalline materials, Adv. Phys. 18 (1969) 41-102, http://dx.doi.org/10.1080/00018736900101267.

[26] Y.A. Firsov, Small Polarons: transport phenomena, Polarons Adv. Mater. (2007) 63. A.S. Alexandrov.

[27] D. Emin, Polarons, Cambridge University Press, New York, 2013.

[28] C. Merschjann, M. Imlau, H. Brüning, B. Schoke, S. Torbrügge, Nonexponential relaxation dynamics of localized carrier densities in oxide crystals without structural or energetic disorder, Phys. Rev. B 84 (2011) 52302, http:// dx.doi.org/10.1103/PhysRevB.84.052302.

[29] B. Sturman, E. Podivilov, M. Gorkunov, Origin of stretched exponential relaxation for hopping-transport models, Phys. Rev. Lett. 91 (2003) 176602 http://dx.doi.org/10.1103/PhysRevLett.91.176602.

[30] J. Carnicero, M. Carrascosa, G. García, F. Agulló-López, Site correlation effects in the dynamics of iron impurities $\mathrm{Fe}^{2+} / \mathrm{Fe}^{3+}$ and antisite defects $\mathrm{Nb}_{\mathrm{Li}}^{4+} / \mathrm{Nb}_{\mathrm{Li}}^{5+}$ after a short-pulse excitation in $\mathrm{LiNbO}_{3}$, Phys. Rev. B 72 (2005) 245108, http:// dx.doi.org/10.1103/PhysRevB.72.245108.

[31] A. Miller, E. Abrahams, Impurity conduction at low concentrations, Phys. Rev. 120 (1960) 745-755, http://dx.doi.org/10.1103/PhysRev.120.745.
[32] J. Schnakenberg, Polaronic impurity hopping conduction, Phys. Status Solidi B 28 (1968) 623-633, http://dx.doi.org/10.1002/pssb.19680280220.

[33] A. Zylbersztejn, Thermally activated trapping in Fe-doped $\mathrm{LiNbO}_{3}$, Appl. Phys Lett. 29 (1976) 778-780, http://dx.doi.org/10.1063/1.88946.

[34] A. Dhar, A. Mansingh, Optical properties of reduced lithium niobate single crystals, J. Appl. Phys. 68 (1990) 5804-5809, http://dx.doi.org/10.1063 1.346951

[35] S. Mamoun, A.E. Merad, L. Guilbert, Energy band gap and optical properties of lithium niobate from ab initio calculations, Comput. Mater. Sci. 79 (2013) 125-131, http://dx.doi.org/10.1016/j.commatsci.2013.06.017.

[36] I.I. Fishchuk, On the theory of hopping transport in organic solids with superimposed disorder and polaron effects, Philos. Mag. Part B 81 (2001) 561-568, http://dx.doi.org/10.1080/13642810108225451.

[37] K. Peithmann, A. Wiebrock, K. Buse, Photorefractive properties of highlydoped lithium niobate crystals in the visible and near-infrared, Appl. Phys. B 68 (1999) 777-784, http://dx.doi.org/10.1007/s003400050704.

[38] H.H. Nahm, C.H. Park, First-principles study of microscopic properties of the $\mathrm{Nb}$ antisite in $\mathrm{LiNbO}_{3}$ : comparison to phenomenological polaron theory, Phys. Rev. B 78 (2008) 184108, http://dx.doi.org/10.1103/PhysRevB.78.184108. 